\title{
Design and Measurements of a High-Performance Wideband Transmitarray Antenna for D-band Communications
}

\author{
Wassim Saleh, Yoann Letestu, Ronan Sauleau, Fellow, IEEE, and Eduardo Motta Cruz
}

\begin{abstract}
A low-cost dual linearly-polarized transmitarray antenna (TA) is presented for $5 \mathrm{G}$ and beyond networks at D-band. This TA is made of $40 \times 40$ unit-cells (UCs) and is illuminated by a stepped feed horn with a F/D ratio equal to 0.75 . The flat lens panel is based on UCs with six different phase states at the center frequency of $150 \mathrm{GHz}$ (thus leading in average to a 2-bit phase quantization and 0.7-dB insertion loss from 130 to $175 \mathrm{GHz}$ ). Each $\mathrm{UC}$ is made of only three metal layers and does not include any via connection to guaranty performance robustness with respect to fabrication dispersions. The measured 1-dB and 3-dB TA gain bandwidths equal $16.8 \%$ and $19.8 \%$, respectively, with a peak gain of $32 \mathrm{dBi}$, corresponding to an aperture efficiency of $32 \%$ at $150 \mathrm{GHz}$. The measured sidelobe and cross-polarization levels in $E$ and $H$ planes remain below -21 and $-37 \mathrm{~dB}$, respectively. An excellent agreement between measured and simulated radiation patterns was observed.
\end{abstract}

Index Terms-Transmitarray, D-band, dual polarization, millimeter-wave antenna, backhauling, high gain.

\section{INTRODUCTION}

$\mathbf{T}$ HE growth of mobile data traffic is driving continuous innovation in wireless communications, and the main challenges in next-generation mobile networks (5G and beyond) require increased capacity of data traffic (up to 100 Gbps) with extremely low latency. Due to its large bandwidth available, the D-band (130-174.8 GHz) is now being considered as a potential candidate for high-capacity millimeter-wave links, especially for small cell backhauling in urban point-topoint networks [1-4]. Since there are no substantial differences between the $\mathrm{V}$ and $\mathrm{H}$ polarizations in the propagation system gain of the D-band network, the use of dual-polarization antenna systems is advantageous to reduce the number of antennas and their footprint on site, with frequency reuse.

In this context, the use of Printed Circuit Board (PCB) TAs is a relevant choice, thanks to their high gain, absence of feed blockage, low cost and possible simplicity of construction.

This work has been done under a French research program CIFRE (Convention Industrielle de Formation par la REcherche), between IETR (Institut d'Electronique et des Technologies du numéRique) and RFS (Radio Frequency Systems) company.

W. Saleh is with IETR UMR CNRS 6164, Université de Nantes, Polytech Nantes, Rue Christian Pauc, 44306 Nantes, France and Radio Frequency Systems, Rue J. B. Marcet, Trignac, France (email Wassim.saleh@univ-nantes.fr). Y. Letestu is with Radio Frequency Systems, Rue J. B. Marcet, Trignac, France (email: Yoann.Letestu@rfsworld.com; andre.doll@rfsworld.com).

R. Sauleau is with Univ Rennes, CNRS, IETR - UMR 6164, F-35000 Rennes, France (email: ronan.sauleau@univ-rennes1.fr).

E. Motta Cruz is with IETR UMR CNRS 6164, Université de Nantes, Polytech Nantes, Rue Christian Pauc, 44306 Nantes, France (email : eduardo.mottacruz@univ-nantes.fr).
Nonetheless most of the solutions existing at millimeterwaves operate mainly in single-polarization and suffer from moderate bandwidths $[5,6]$ or use a large number of dielectric layers [7,8] (more than 5) and metallized via holes [9-11]. A broadband three-layer element is presented in $[12,13]$; it is formed by two orthogonal metallic polarizers separated by two identical substrates. However, this very promising solution works in a single polarization only, which limits their application.

There have been only a few studies on dual-polarized TA configurations in the literature. Two types of TA composed of aperture-coupled microstrip UCs with 3 metallic layers have been proposed in [14-15], with 1-dB gain bandwidths between 18 and $20 \%$, but their cross-polarization discrimination is not provided. A TA using a dual-polarized element with Jerusalem cross slot is introduced at W-band in [16], but no experimental demonstration is provided in this work. In [17] a TA with four metal layers and three air gaps was studied, with a 1-dB gain bandwidth of $12.6 \%$ at $11.7 \mathrm{GHz}$, nevertheless this concept of the stacked layers configuration is not suitable for Dband communications due to its manufacturing and assembly complexity. Moreover, a dual-polarized TA with a 1-dB gain bandwidth of $16 \%$ and a cross-polarization level of $-22 \mathrm{~dB}$ was described in [18], but this TA configuration also suffers from a complex architecture characterized by a pair of tri-layer frequency selective surfaces separated by a quarter-wavelength air gap.

In this communication, we present a dual-polarized TA at D-band with a broadband operation of $19.8 \%$, a maximum gain of $32 \mathrm{dBi}$, and a high cross-polarization isolation (below $-37 \mathrm{~dB}$ ). This paper is organized as follows. The geometry of the UC is given in section II. The TA design (including the feeding source) as well as the simulation and measurement results are discussed in section III . Finally, the conclusions are drawn in section IV.

\section{DUAL-POLARIZED UNIT-CELL}

The proposed UC has a size of $1 \times 1 \mathrm{~mm}^{2}\left(\lambda_{0} / 2 \times \lambda_{0} / 2\right)$, where $\lambda_{0}$ is the wavelength in free space at the central frequency $(150 \mathrm{GHz})$. The UC stack-up and topology have been selected by considering two major design-driving constraints: first to allow the use of standard PCB manufacturing techniques (minimum line and gap widths of $100 \mu \mathrm{m}$ ), and second to avoid metallized-via connection so as to reduce the TA fabrication cost and improve its robustness with respect to 
manufacturing dispersions. This stack-up, represented in Fig. $1 \mathrm{a}$, is made of three metallic layers separated by two Rogers 5880 substrates $\left(\varepsilon_{\mathrm{r}}=2.2, \tan \delta=0.0036\right)$ and assembled by a $114-\mu \mathrm{m}$ thick FR-28 bonding film $\left(\varepsilon_{\mathrm{r}}=2.72, \tan \delta=0.0056\right)$.

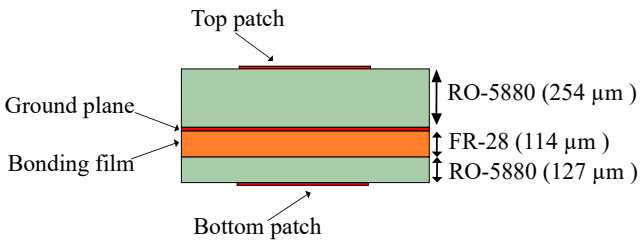

(a)

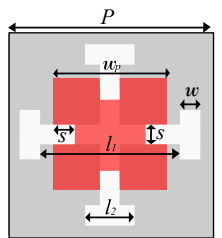

(b) $\mathrm{UC} 1-45^{\circ}$

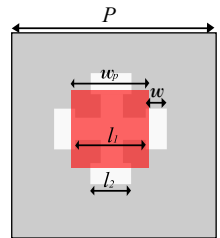

(e) UC4 - $180^{\circ}$

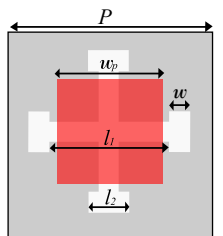

(c) UC2 $-90^{\circ}$

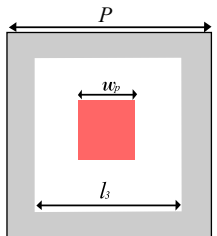

(f) UC5 - $225^{\circ}$

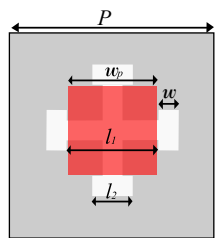

(d) UC3 - $135^{\circ}$

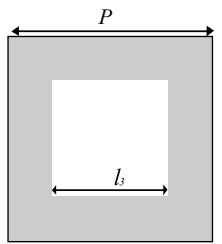

(g) UC6 - $270^{\circ}$
Fig. 1. UC geometry: (a) Dielectric stack-up; (b) UC1; (c) UC2;(d) UC3; (e) UC4; (f) UC5; (g) UC6. The ground plane is depicted in gray.

In contrast to Refs. [19-21], the geometry of the proposed UC is fully symmetric and operates in dual polarization, thus leading to a high level of cross-polarization discrimination. To operate over a broadband and provide simultaneously an equivalent phase quantization of about 2 bits, 6 different UCs, UC1 to UC6, are used here. They have been designed to exhibit phase shift values equal to $45^{\circ}, 90^{\circ}, 135^{\circ}, 180^{\circ}, 225^{\circ}$ and $270^{\circ}$ at $150 \mathrm{GHz}$, respectively, their geometry is provided in Figs. 1b-1g.

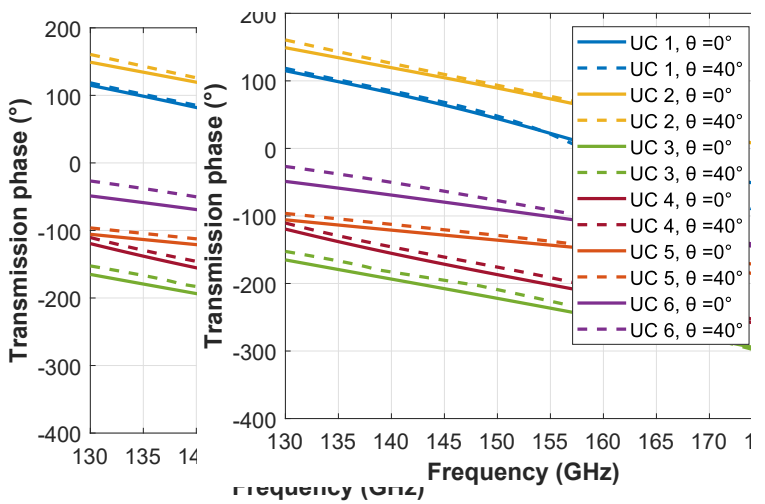

Fig. 2. Simulated phase of the transmission coefficient for each unit cell, under normal and oblique incidence.

The frequency responses of these UCs have been computed and optimized using Ansys HFSS version 2020 with periodic boundary conditions and Floquets ports. Their scattering parameters are provided in our previous study [23]. Here we
TABLE I

MAIN UC GEOMETRIC PARAMETERS

\begin{tabular}{ccc}
\hline \hline Design & Cell & Dimensions $(\mathrm{mm})$ \\
\hline Fig. 2b & UC1 & $\mathrm{P}=1, w_{p}=0.555, w=0.1, l_{1}=0.7, l_{2}=0.25, \mathrm{~s}=0.1$ \\
\hline Fig. 2c & UC2 & $\mathrm{P}=1, w_{p}=0.52, w=0.1, l_{1}=0.6, l_{2}=0.2$ \\
\hline Fig. 2d & UC3 & $\mathrm{P}=1, w_{p}=0.39, w=0.1, l_{1}=0.35, l_{2}=0.2$ \\
\hline Fig. 2e & UC4 & $\mathrm{P}=1, w_{p}=0.44, w=0.1, l_{1}=0.45, l_{2}=0.2$ \\
\hline Fig. 2f & UC5 & $\mathrm{P}=1, w_{p}=0.29, l_{3}=0.8$ \\
\hline Fig. 2g & UC6 & $\mathrm{P}=1, l_{3}=0.57$ \\
\hline \hline
\end{tabular}

only provide the phase responses of the $6 \mathrm{UCs}$ computed under normal and oblique incidence $\left(40^{\circ}\right)$ between 130 and $175 \mathrm{GHz}$ (Fig. 2). Additional results (not reported here) have shown that the additional insertion loss under oblique incidence remains lower than $1 \mathrm{~dB}$ across the entire frequency band.

The equivalent number of phase quantization bits and the average value of insertion loss are plotted in Fig. 3 as a function of frequency. The equivalent quantization is calculated by considering the phase difference between two consecutive states, as defined by [22]. Here we can appreciate the highperformance of the proposed UCs with average values of equivalent quantization and insertion loss respectively equal to 2 bits and $0.7 \mathrm{~dB}$ between 130 and $175 \mathrm{GHz}$ (Fig. 3).

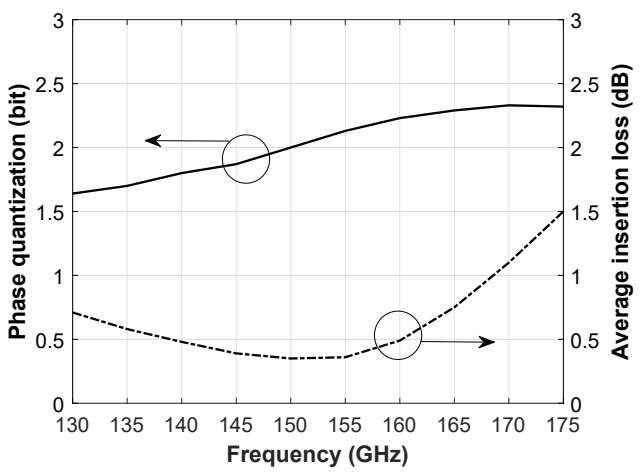

Fig. 3. Number of equivalent bits and average insertion loss as a function of frequency for UCs.

\section{TRANSMITARRAY: DESIGN AND PROTOTYPING}

\section{A. Array design and focal source}

A dual-polarized TA based on the proposed UC has been designed and fabricated using standard PCB technology. The total aperture size of the final prototype including the mechanical frame is $60 \times 60 \mathrm{~mm}^{2}$ (Fig. 4), with an effective radiating area of $40 \times 40 \mathrm{~mm}^{2}\left(20 \lambda_{0} \times 20 \lambda_{0}\right)$.

The TA is illuminated by a linearly-polarized stepped horn, placed at a distance of $30 \mathrm{~mm}$ from the TA panel (corresponding to a F/D ratio of 0.75 and an edge taper of $10 \mathrm{~dB}$ ). The inner walls of the TA mechanical support are coated by a $3 \mathrm{~mm}$ thick carbon-based foam layer to reduce internal reflections on the outer mechanical frame.

The aperture size of the feed horn equals $3.68 \mathrm{~mm} \times 2.86$ $\mathrm{mm}$. It incorporates a transition to WR6 waveguide and is made of seven steps. It has been designed and optimized using 
Ansys HFSS to minimize its reflection coefficient (Fig. 5a) and simultaneously provide the desired edge taper illumination; the slight differences observed between the measured and data are attributed to fabrication uncertainties.

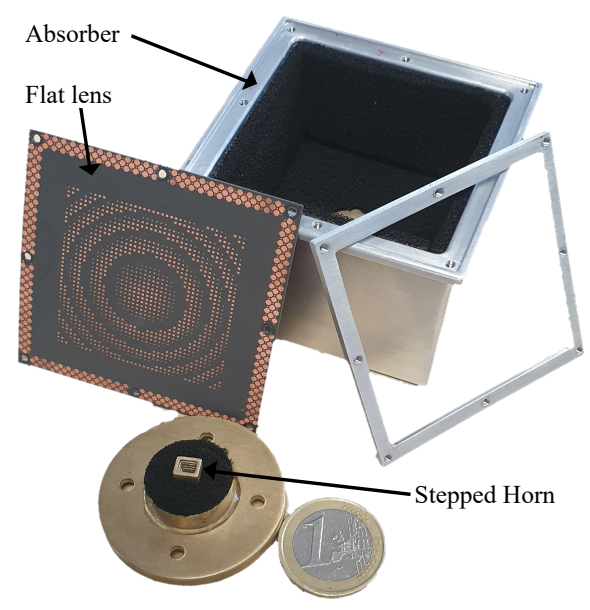

Fig. 4. Fabricated TA prototype.

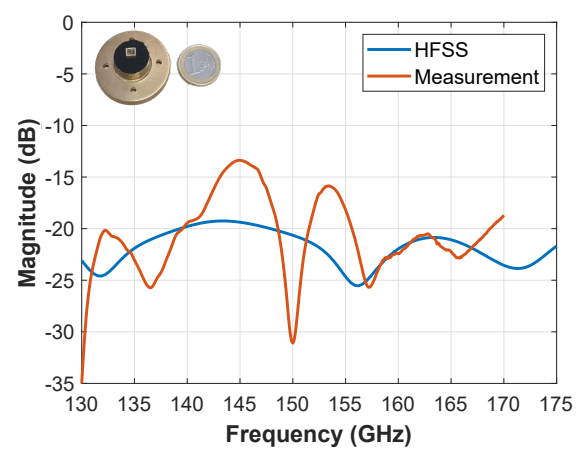

(a)

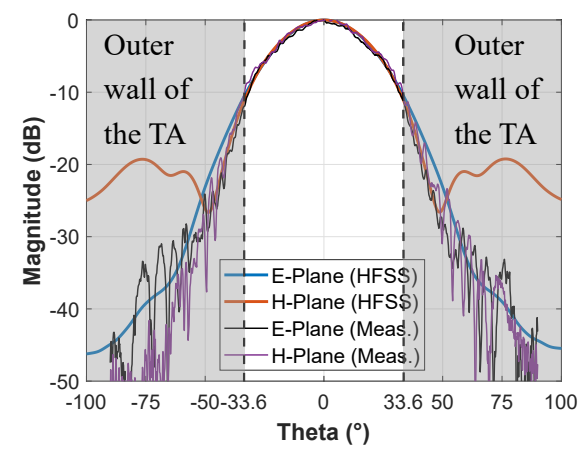

(b)

Fig. 5. Simulated and measured performance of the stepped feed horn: (a) Reflection coefficient $S_{11}$; (b) Co-polarized radiation patterns in E- and $\mathrm{H}$ planes at $150 \mathrm{GHz}$.

At $150 \mathrm{GHz}$, this feed horn exhibits a maximum measured gain of $13 \mathrm{dBi}$ and a nearly circularly-symmetric $\operatorname{cosq}(\theta)$ pattern (with $\mathrm{q}=5$ ), as shown in Fig. 5b. The agreement between measurements and simulations is excellent for elevation angles $<45^{\circ}$ (this value is larger than the maximum incidence angle on the TA panel).

\section{B. Performance of the TA prototype}

The full-wave simulations have been carried out using the Finite-element Boundary-Integral (FE-BI) solver available in Ansys HFSS version 2020. The numerical model includes the stepped feed horn and the TA panel but do not take into account the mechanical outer frame shown in Fig. 4. The phase distribution has been calculated and optimized at $150 \mathrm{GHz}$ to maximize the aperture efficiency and obtain a low side lobe level (SLL) by selecting the most relevant UC at the center of the TA panel (namely UC2). The optimal phase compensation law on the flat lens aperture is plotted in Fig. 6a; its prototyped version is represented in Fig. $6 b$.

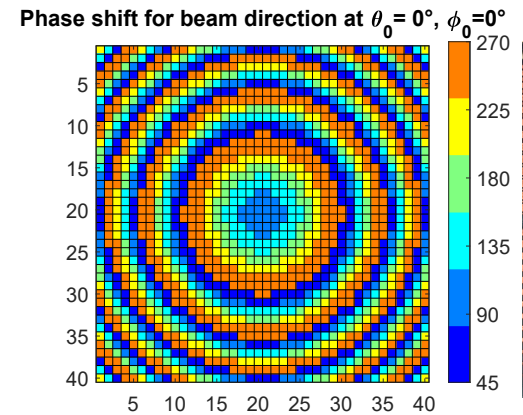

(a)

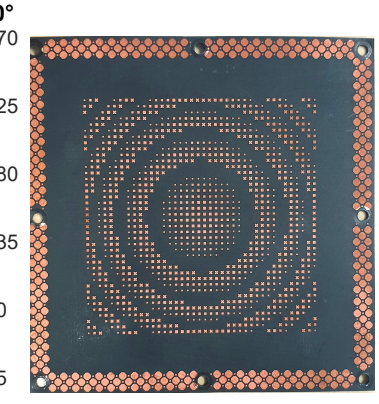

(b)
Fig. 6. TA composed of 40 x 40 UCs: (a) Simulated phase distribution at $150 \mathrm{GHz}$. (b) Top view of the fabricated array.

The simulated and measured reflection coefficients are represented in Fig. 7. The measured data remain lower than -13 $\mathrm{dB}$ between 130 and $175 \mathrm{GHz}$, corresponding to a $-10 \mathrm{~dB}$ fractional bandwidth larger than $30 \%$.

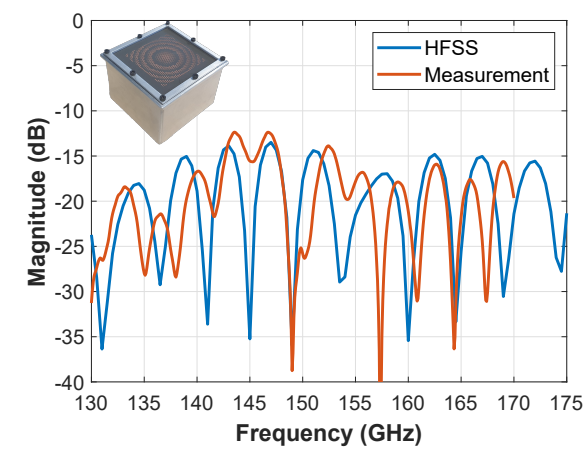

Fig. 7. Simulated and measured reflection coefficients $S_{11}$ of the TA.

The radiation performance of the TA prototype has been characterized in an anechoic chamber using a near-field scanner (Fig. 8). The normalized co- and cross-polarized components are plotted in Fig. 9 in E- and H-planes at 140, 150 and $170 \mathrm{GHz}$. An excellent agreement is observed between fullwave simulations and measurements. The side lobe levels in Eand $\mathrm{H}$-planes remain below $-21 \mathrm{~dB}$ over the whole frequency band of interest; the half-power beam width (HPBW) varies between $3.8^{\circ}$ and $3.1^{\circ}$. The cross-polarization level, also plotted in Fig. 9, is better than $-37 \mathrm{~dB}$. The measurement in the second orthogonal polarization has not carried out, but it can be assumed that the radiation patterns are identical due to the total symmetry of the structure. 


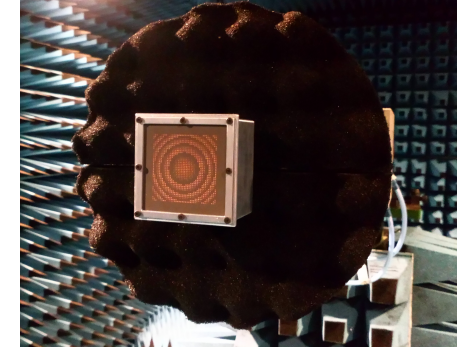

Fig. 8. TA antenna prototype under test in the anechoic chamber.
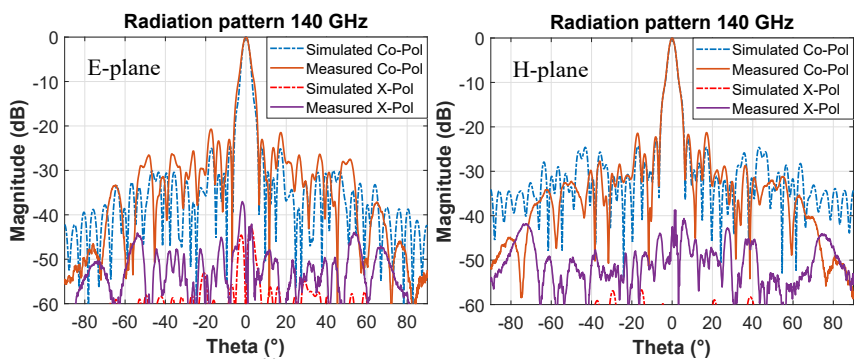

(a)
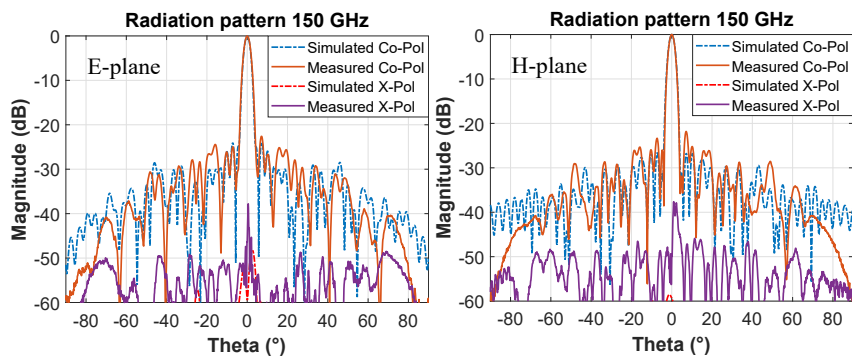

(b)
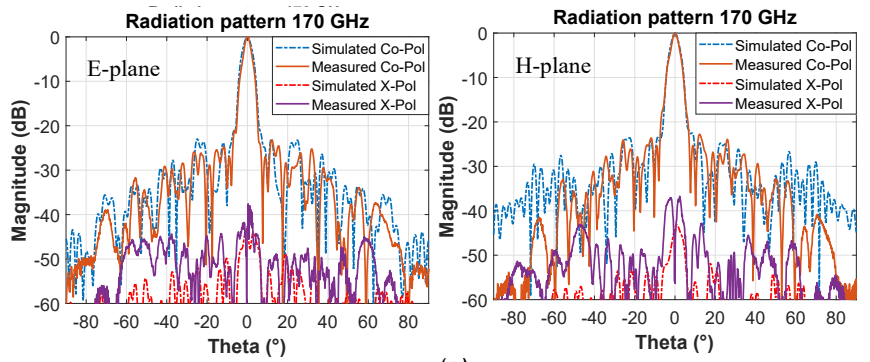

(c)

Fig. 9. Simulated and measured co- and cross-polarized radiation patterns of the TA prototype: (a) $140 \mathrm{GHz}$; (b) $150 \mathrm{GHz}$; (c) $170 \mathrm{GHz}$.

The antenna gain has been measured by comparison with a standard gain horn [24], Fig. 10. Its maximum value reaches 32 $\mathrm{dBi}$ at $150 \mathrm{GHz}$ (which corresponds to an aperture efficiency of $32 \%$ ) and its $3-\mathrm{dB}$ gain bandwidth equals $19.8 \%$.

The measured gain value agrees well with the simulated results, taking into account the systematic losses coming from the machining error and roughness of the of the primary source material (between 1 and $2 \mathrm{~dB}$ over the whole bandwidth), with a slight shift towards the higher frequencies. This slight gain deviation is attributed to etching uncertainties $( \pm 30 \mu \mathrm{m})$.

Table II compares the main performance of the proposed TA with others prototypes of the literature and operating at D-band. To the best of our knowledge, we report here the first broadband ( $\sim 20 \%)$ high-gain $(>30 \mathrm{dBi})$ dual-polarized

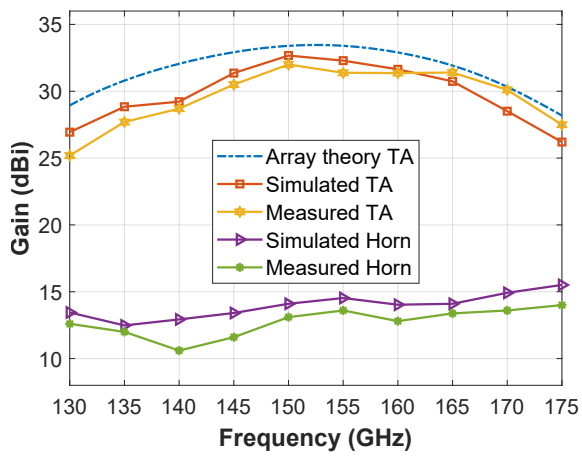

Fig. 10. Measured and simulated gain as a function of frequency.

TA. The TA reported in Ref. [9] exhibits similar radiation performance but operates in single polarization and is based on a more complex dielectric stack-up since in integrates metallic via holes. Ref. [11] describes a TA prototype in LTCC with a much larger number of metallic layers (7 instead of 3 ) and a more complex fabrication stream.

TABLE II

COMPARISON OF THE PROPOSED TRANSMITARRAY WITH OTHER WORKS AT MM-WAVES

\begin{tabular}{cccc}
\hline \hline Ref & This work & {$[9]$} & {$[11]$} \\
\hline Freq $f_{0}(\mathrm{GHz})$ & 150 & 145 & 140 \\
\hline Technology & PCB & PCB & LTCC \\
\hline Polarization & DUAL LP & LP & LP \\
\hline Vias & No & Yes & Yes \\
\hline N. metal layer & 3 & 3 & 7 \\
\hline Aperture size & $4 \mathrm{~cm}^{2}$ & $4.2 \mathrm{~cm}^{2}$ & $4 \mathrm{~cm}^{2}$ \\
\hline Peak measured Gain $(\mathrm{dBi})$ & 32 & 33 & 33.5 \\
\hline Max. aperture efficiency & $32 \%$ & $38.3 \%$ & $30.8 \%$ \\
\hline 3 dB-gain BW & $19.8 \%$ & $19.8 \%$ & $24.4 \%$ \\
\hline F/D & 0.75 & 0.75 & 1.87 \\
\hline XPD $(\mathrm{dB})$ at $f_{0}$ & -37 & -30 & -31 \\
\hline SLL $(\mathrm{dB})$ at $f_{0}$ & -21 & -21 & -21 \\
\hline \hline
\end{tabular}

\section{CONCLUSION}

A dual-polarized 1600 elements TA, based on a low-cost PCB technology with only three metal layers and no metalized via hole, has been designed and characterized at D-band. Its uses six different unit-cell configurations to reach a nearly 2bit phase resolution over a $45-\mathrm{GHz}$ bandwidth. The theoretical results obtained by array theory and full-wave simulations have been validated experimentally. The aperture size of the TA prototype equals $40 \times 40 \mathrm{~mm}^{2}$; it is illuminated by a $13-\mathrm{dBi}$ gain stepped horn. The measured TA peak gain reaches 32 $\mathrm{dBi}$ with an aperture efficiency of $32 \%$. Its $1-\mathrm{dB}$ and $3-\mathrm{dB}$ gain bandwidths are $16.8 \%$ and $19.8 \%$ respectively. Within the operating frequency band, its measured side lobe level and cross-polarization in both planes $\mathrm{E}$ and $\mathrm{H}$ remains below -21 and $-37 \mathrm{~dB}$, respectively.

\section{ACKNOWLEDGMENT}

The authors thank Robert STARAJ and Laurent BROCHIER at LEAT (Laboratory of Electronics, Antennas and Telecommunications) team at Sophia Antipolis (Nice, France) for carrying out of the antenna measurements. 


\section{REFERENCES}

[1] M. Jaber, M. A. Imran, R. Tafazolli, and A. Tukmanov, "5G Backhaul Challenges and Emerging Research Directions: A Survey," IEEE Access, vol. 4. Institute of Electrical and Electronics Engineers Inc., pp. 1743-1766, 2016.

[2] ETSI, "ETSI GR mWT 018 - V1.1.1 - Analysis of Spectrum, License Schemes and Network Scenarios in the W-band," vol. 1, pp. 1-26, 2019.

[3] ECC, "Point-to-Point Radio Links in the Frequency Ranges 92-114.25 GHz and 130-174.8 GHz," no. September, 2018.

[4] "Aspects of microwave transport evolution - the Nokia update," pp. $1-26$.

[5] F. Diaby, A. Clemente, L. Di Palma, L. Dussopt, and R. Sauleau, "Impact of phase compensation method on transmitarray performance," 2017 11th Eur. Conf. Antennas Propagation, EUCAP 2017, pp. 3114-3118, 2017.

[6] S. L. Liu, X. Q. Lin, Z. Q. Yang, Y. J. Chen, and J. W. Yu, "WBand Low-Profile Transmitarray Antenna Using Different Types of FSS Units," IEEE Trans. Antennas Propag., vol. 66, no. 9, pp. 4613-4619, Sep. 2018.

[7] A. H. Abdelrahman, P. Nayeri, A. Z. Elsherbeni, and F. Yang, "Bandwidth Improvement Methods of Transmitarray Antennas," IEEE Trans. Antennas Propag., vol. 63, no. 7, pp. 2946-2954, Jul. 2015.

[8] A. E. Mahmoud, W. Hong, Y. Zhang, and A. Kishk, "W-band mutlilayer perforated dielectric substrate lens," IEEE Antennas Wirel. Propag. Lett., vol. 13, no. January, pp. 734-737, 2014.

[9] F. Foglia Manzillo, A. Clemente, and J. L. González-Jiménez, "HighGain D-Band Transmitarrays in Standard PCB Technology for Beyond5G Communications," IEEE Trans. Antennas Propag., vol. 68, no. 1, pp. 587-592, Jan. 2020.

[10] C. Jouanlanne et al., "Wideband Linearly Polarized Transmitarray Antenna for $60 \mathrm{GHz}$ Backhauling," IEEE Trans. Antennas Propag., vol. 65 , no. 3, pp. 1440-1445, 2017.

[11] Z. W. Miao et al., "140 GHz High-Gain LTCC-Integrated TransmitArray Antenna Using a Wideband SIW Aperture-Coupling Phase Delay Structure," IEEE Trans. Antennas Propag., vol. 66, no. 1, pp. 182-190, 2018.

[12] J. Yin, Y. Zhang, G. Deng, R. Chen, and H. Chen, "Ultra-wideband transmitarray antennas design based on ultrathin polarization conversion elements," Asia-Pacific Microw. Conf. Proceedings, APMC, vol. 2019-Decem, pp. 423-425, 2019.

[13] P. Y. Feng, S. W. Qu, and S. Yang, "Octave Bandwidth Transmitarrays with a Flat Gain,” IEEE Trans. Antennas Propag., vol. 66, no. 10, pp. 5231-5238, Oct. 2018.

[14] K. Pham et al., "Design of Wideband Dual Linearly Polarized Transmitarray Antennas," IEEE Trans. Antennas Propag., vol. 64, no. 5, pp. 2022-2026, 2016.

[15] X. Yang, Y. Zhou, L. Xing, and Y. Zhao, "A wideband and low-profile transmitarray antenna using different types of unit-cells," Microw. Opt. Technol. Lett., vol. 61, no. 6, pp. 1584-1589, Jun. 2019.

[16] X. Dong, Y. Xiao, and H. Sun, "Design of a W-band 3-bit Dual Linearly Polarized Transmit-array Antenna," 2018 12th Int. Symp. Antennas, Propag. EM Theory, ISAPE 2018 - Proc., no. c, pp. 1-3, 2019.

[17] C. Y. Hsu, L. T. Hwang, T. S. Horng, S. M. Wang, F. S. Chang, and C. N. Dorny, "Transmitarray design with enhanced aperture efficiency using small frequency selective surface cells and discrete jones matrix analysis," IEEE Trans. Antennas Propag., vol. 66, no. 8, pp. 3983-3994, 2018.

[18] Q. Luo, S. Gao, M. Sobhy, and X. Yang, "Wideband Transmitarray with Reduced Profile,' IEEE Antennas Wirel. Propag. Lett., vol. 17, no. 3, pp. 450-453, Mar. 2018.

[19] M. Smierzchalski, A. Clemente, M. Huchard and C. Barbier, "Wideband low-profile transmitarray antenna for backhauling at $60 \mathrm{GHz}$," 12th European Conference on Antennas and Propagation (EuCAP 2018), 2018, pp. 1-5.

[20] L. Dussopt et al., "A V-Band Switched-Beam Linearly Polarized Transmit-Array Antenna for Wireless Backhaul Applications," IEEE Trans. Antennas Propag., vol. 65, no. 12, pp. 6788-6793, Dec. 2017.

[21] L. Dussopt, A. Moknache, T. Potelon, and R. Sauleau, "Switched-beam E-band transmitarray antenna for point-to-point communications," 2017 11th Eur. Conf. Antennas Propagation, EUCAP 2017, pp. 3119-3122, 2017.

[22] R. Pereira, R. Gillard, R. Sauleau, P. Potier, T. Dousset, and X. Delestre, "Dual linearly-polarized unit-cells with nearly 2-bit resolution for reflectarray applications in X-band," IEEE Trans. Antennas Propag., vol. 60 , no. 12 , pp. 6042-6048, 2012.
[23] W. Saleh, Y. Letestu, R. Sauleau, and E. M. Cruz, "High-gain Transmitarray Antenna for Backhauling at D-band," 15th Eur. Conf. Antennas Propagation, EuCAP 2021, pp. 31-34, 2021.

[24] "Spherical Near-Field Scanner Systems." [Online]. Available: https://www.nsi mi.com/products/system-solutions/near-fieldsystems/spherical-near-field-scanner-systems. [Accessed: 04-Jun2021].

\section{ACCEPTED MANUSCRIPT}

